\title{
Frontières
}

\section{La « bonne mort " : une durée idéale pour la fin de vie en unité de soins palliatifs?}

\section{Florence Ollivier}

Volume 20, numéro 1, automne 2007

La « bonne mort »

URI : https://id.erudit.org/iderudit/017945ar

DOI : https://doi.org/10.7202/017945ar

Aller au sommaire du numéro

Éditeur(s)

Université du Québec à Montréal

ISSN

1180-3479 (imprimé)

1916-0976 (numérique)

Découvrir la revue

Citer cet article

Ollivier, F. (2007). La « bonne mort » : une durée idéale pour la fin de vie en unité de soins palliatifs ? Frontières, 20(1), 34-39.

https://doi.org/10.7202/017945ar
Résumé de l'article

Nous nous intéresserons dans cet article à la dimension temporelle de la " bonne mort " en unité de soins palliatifs. L'institution, en raison de son organisation rationalisée du travail, produit une norme temporelle d'environ 21 jours, en moyenne. Il ne s'agit, bien entendu que d'une moyenne, mais elle suggère une durée optimale au cours de laquelle le patient est invité à cheminer et à préparer sa propre mort. L'article souligne comment les attentes de l'institution peuvent être troublées par la présence de patients trop pressés de mourir, et qui ne semblent pas déterminés à attendre l'échéance biologique. Enfin, l'article décrit la manière dont l'institution s'efforce d'adapter ses usagers à cette durée idéale. 


\section{Résumé}

Nous nous intéresserons dans cet article à la dimension temporelle de la «bonne mort» en unité de soins palliatifs. L'institution, en raison de son organisation rationalisée du travail, produit une norme temporelle d'environ 21 jours, en moyenne. II ne s'agit, bien entendu que d'une moyenne, mais elle suggère une durée optimale au cours de laquelle le patient est invité à cheminer et à préparer sa propre mort. L'article souligne comment les attentes de l'institution peuvent être troublées par la présence de patients trop pressés de mourir, et qui ne semblent pas déterminés à attendre l'échéance biologique. Enfin, l'article décrit la manière dont l'institution s'efforce d'adapter ses usagers à cette durée idéale.

Mots clés: mort - soins palliatifs management - usagers - France.

\section{Abstract}

In this article, we will be looking at the temporal dimension of the "good death" as defined in palliative care units. The institution of palliative care produces a temporal norm of about 21-days on average because of labour rationalization. This is, of course, only an average, but it implies an implicit optimum duration during which the patient is led to prepare his own death. This article underlines how the institution's expectations may be at odds with patients who are too eager to die, and who do not seem to be willing to wait for the end of the biological term. Finally, the article describes the way the institution strives to have patients adapt to this ideal duration.

Keywords: dying process - palliative care units - management - patients France.

$\begin{array}{lllllllll}\text { A } & \mathrm{R} & \mathrm{T} & \mathrm{I} & \mathrm{C} & \mathrm{L} & \mathrm{E} & \mathrm{S}\end{array}$

\section{LA «BONNE MORT» Une durée idéale pour la fin de vie en unité de soins palliatifs?}

\author{
Florence Ollivier ${ }^{1}$, \\ doctorante en sociologie,
}

Laboratoire Cultures et sociétés urbaines - Paris VIII.

Depuis les années 1960, les publications au sujet de la mort se multiplient ${ }^{2}$, dénonçant souvent l'isolement des mourants à l'hôpital. À la même époque, les premières pratiques des soins palliatifs se développent dans les pays anglo-saxons grâce aux recherches de Cicely Saunders, en Angleterre, sur la douleur et à celles de la psychiatre Elisabeth Kübler-Ross (1969) sur la vie psychique des patients à l'approche de leur mort. Leurs apports théoriques ne tardent pas à être importés ailleurs.

En France, les soins palliatifs prennent leur essor, un peu plus tard, avec la mise en place de la première unité de soins palliatifs, à Paris, en 1985. La circulaire Laroque $^{3}$, en 1986, ouvre ensuite la voie à une reconnaissance institutionnelle de la pratique des soins palliatifs; le mourant est invité à prendre conscience de sa propre mort, à se confier et à se soulager de ses douleurs morales: il s'agit de replacer le patient au cœur des pratiques de soins de manière à satisfaire au mieux ses besoins. Ce projet se traduit aussi par la volonté de s'affranchir des contraintes économiques inhérentes à la pratique médicale. Les pionniers des soins palliatifs déclaraient ainsi dans la circulaire Laroque: «les soins palliatifs ne sauraient en aucun cas se concrétiser par une médecine à un moindre coût». Pour autant, le dernier rapport de la Cour des comptes révèle, dès 2003, une baisse conséquente du financement alloué aux structures des soins palliatifs ${ }^{4}$. Il convient donc de voir comment l'institution des soins palliatifs concilie l'idéal de son projet avec les contraintes structurelles auxquelles elle est, aujourd'hui, soumise.

Notre méthodologie ${ }^{5}$ croise entretiens et observations des pratiques d'accompagnement en unité de soins palliatifs. Le choix de l'observation repose sur deux raisons essentielles. D'une part, elle nous apparaît être la méthode la plus efficace pour travailler sur notre objet. En effet, la pratique en soins palliatifs est difficilement transmissible par le biais des entretiens et la mort demeure un objet trop intime et suffisamment empli de souffrances pour qu'il soit dévoilé lors du premier passage d'une étrangère. D'autre part, les pionniers des soins palliatifs ont construit leur légitimité sur des travaux de recherches basés essentiellement sur des observations dans des services hospitaliers et des entretiens 
auprès de malades. Les travaux d'Elisabeth Kübler-Ross s'appuient sur un long travail de terrain et d'observation (1969), de même que les travaux, qui lui sont contemporains, des sociologues A. Strauss et B. Glaser $(1965,1968)$ sur le traitement de la mort à l'hôpital. La culture palliative est donc, par cette tradition de recherche, plutôt favorable à la présence d'une observatrice. Pour ces deux raisons, les données sur lesquelles nous nous appuierons proviennent essentiellement d'un travail ethnographique conduit, en France, entre février et avril 2005 dans deux unités de soins palliatifs de la région parisienne ${ }^{6}$. J'ai été intégrée au quotidien du travail des médecins, des soignants et de la psychologue. Ainsi, j'ai assisté aux réunions des transmissions revêtue d'une blouse blanche, j'ai observé les consultations avec les médecins, j'étais présente lors de l'évaluation des douleurs, j'ai participé aux repas avec les soignantes et j'ai suivi la psychologue lors de ses visites. Je passais régulièrement quatre à cinq journées par semaine, de jour comme de nuit, dans le service, ce qui m'a permis de suivre 42 trajectoires de patients en fin de vie.

\section{COMMENT CONCILIER L'IDÉAL D'HUMANISATION DES SOINS AVEC LES CONTRAINTES STRUCTURELLES?}

Selon Herzlich, un consensus social s'est formé autour de l'idée selon laquelle le patient «a droit à une certaine qualité et dignité de la mort, et à ce qu'elle ne soit pas escamotée»(Herzlich, 1976, p. 198). Pour autant, selon le même auteur, l'institution des soins palliatifs, au même titre que n'importe quel autre service de soins, n'échappe pas à la restriction budgétaire, et par là à la rationalisation du travail et du temps. Il semble alors probable que l'administration du processus biologique de l'agonie soit aussi tributaire, dans une certaine mesure, du cadre organisationnel du service hospitalier. Comme les acteurs des soins palliatifs le rappellent, chaque fin de vie est singulière. Néanmoins, en France, on constate que la durée des trajectoires des fins de vie des patients varie peu, à quelques exceptions près, et elle se conforme à une moyenne de 21 jours dans les différentes structures d'unités de soins palliatifs. Il ne s'agit, bien entendu, que d'une moyenne, et une étude statistique précise montrerait sans doute des variations importantes. Cependant, cette norme temporelle semble marquer le discours des acteurs de l'institution. En effet, à notre première visite dans une des unités de soins palliatifs observées, le chef de service avait évoqué cette norme tempo- relle en la formulant de cette manière: «21 jours, en moyenne ${ }^{7}$.

« 21 jours »: cette durée correspond à une mort, ni trop courte, ni trop longue; une mort d'une durée suffisante pour laisser le temps au patient et à ses proches de cheminer et de se rapprocher au mieux d'une «bonne mort». Cependant, le discours des soignants se heurte à une contradiction: d'un côté, ils cherchent à accompagner la fin de vie d'un patient en respectant son rythme et, de l'autre, ils sont engagés au sein d'une clinique, c'est-à-dire une administration qui «vise à organiser la mort, à en faire un évènement intégrable dans l'activité quotidienne» du service (Herzlich, 1976, p. 205). Dès lors, comment concilier le principe d'humanisation des soins et la pratique institutionnelle soumise à une rationalité économique? Autrement dit, comment l'institution parvient-elle à calquer son rythme de travail sur la temporalité supposée "naturelle» du mourir?

\section{ASSOUPLISSEMENT \\ DE LA STRUCTURE HOSPITALIÈRE ET RYTHME DE TRAVAIL}

En unité de soins palliatifs, l'équipe pluridisciplinaire se compose de soignants, de médecins, d'un kinésithérapeute à mitemps, d'un psychologue à mi-temps et de bénévoles ${ }^{8}$. L'un des objectifs de ces unités consiste à rester au plus près des patients et à accéder à leurs dernières volontés, même les moins raisonnables en apparence. Ainsi en unités de soins palliatifs, il est tout à fait concevable qu'un patient fume malgré son cancer des poumons en phase termi- nale ou qu'un autre organise et célèbre son mariage au sein de l'institution. Un statut privilégié est explicitement attribué aux patients au seuil de la mort, leur ouvrant droit au confort et à une réglementation plus souple: les horaires de visites sont élargis, les proches peuvent se retrouver la nuit, des repas peuvent être organisés en famille, leurs animaux sont tolérés: tout ce que l'hôpital interdit d'ordinaire pour des raisons de sécurité et d'hygiène est concédé en unité de soins palliatifs. Ces interdictions n'auraient plus de sens à l'approche de la mort. Les deux unités de soins palliatifs où nous avons entrepris notre terrain d'observation rendent assez bien compte de la structure et de la pratique des soins palliatifs telles qu'elles sont présentées par Maurice Abiven (1997), dans son ouvrage intitulé: Une mort plus humaine: expérience d'une unité hospitalière de soins palliatifs.

Tous les agents sollicitent du patient son besoin présumé de s'exprimer, de se confier et de se dévoiler pour surmonter ses souffrances. L'institution des soins palliatifs requiert ainsi du patient mourant qu'il partage son expérience avec un auditoire, et pas n'importe lequel: celui des soignants et des médecins. Ces derniers, placés en position d'experts du mourir, participent et veillent au bon déroulement du processus d'agonie. Les professionnels écoutent, certes pour soulager les souffrances des mourants, mais aussi pour adapter et améliorer, par la suite, les protocoles de soins à leurs besoins. Si les usagers des unités de soins palliatifs bénéficient de soins de confort qu'ils régulent eux-mêmes par un

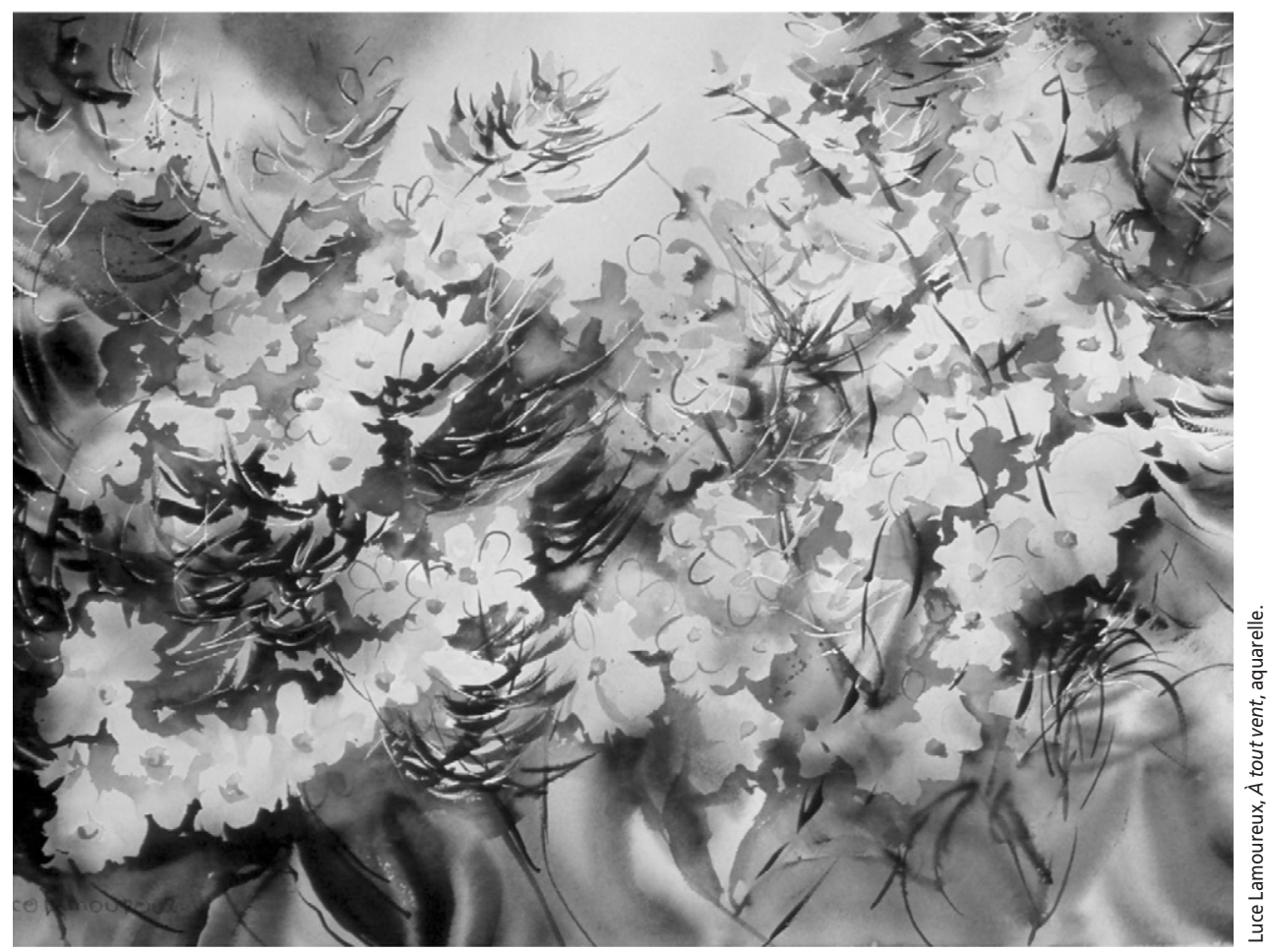


système d'auto-injection, cela ne se fait pas sans une attention a posteriori des soignants aux besoins du patient. Cette demande de justification renvoie à ce que D. Memmi (2003) appelle le "gouvernement par la parole»: il s'agit pour le patient de produire, en contrepartie du soulagement de ses douleurs, un discours autobiographique plus ou moins adapté à l'idéal de la «bonne mort » telle que la définissent généralement les soins palliatifs en France. L'échange de parole permet d'apaiser le patient avant sa mort. Telle serait la dernière étape du cheminement du mourant dans le modèle conceptualisé par la psychiatre Elisabeth Kübler-Ross (1997). Selon ses analyses, l'individu atteint l'état de sublimation ou celui de l'acceptation de la mort après être passé successivement par des étapes préliminaires: la négation, la colère, le marchandage et la dépression.

L'accompagnement individuel du patient dans le travail de cheminement intérieur est en partie le rôle attribué au psychologue de l'unité. Une difficulté demeure: les psychologues essuient des refus dans les services. Parler à une personne cancéreuse en phase terminale ne se fait pas sans difficultés: les métastases cérébrales produisant un ralentissement du débit de la parole et une perte de la mémoire significative, le patient ne maîtrise plus tout à fait ses fonctions cérébrales et physiologiques. Son récit s'effiloche et le vocabulaire se délie considérablement. Ce fut par exemple le cas d'une ancienne ouvrière immigrée du Portugal. Dès son arrivée dans l'unité, elle se montre peu loquace. D'après les soignants, elle est arrivée "trop tard» pour se confier et se préparer à mourir, en raison des séquelles déjà produites par les métastases sur son cerveau. Elle parle plus lentement, cherche ses mots et perd la mémoire des évènements au point de ne plus se souvenir précisément de sa profession. Le psychologue, nous confiant "préférer ceux qui parlent", s'attarde moins dans les chambres des patients «muets». Après deux visites, il abandonne l'espoir de pouvoir faire parler l'ancienne ouvrière : "Laisse, dit-il à son assistante, on n'aura pas mieux ${ }^{9}$.» Le psychologue montre des signes de fatigue et quitte la chambre. D'autres patients plus prolixes l'attendent et le mi-temps accordé par l'institution ne suffit pas pour écouter l'ensemble des usagers. Il est donc amené à concentrer son écoute sur les patients qui peuvent ou qui veulent bien se confier. Son impatience traduit une insatisfaction à ne pas recueillir les paroles de la patiente et le peu de temps de travail qui lui est donné ne place pas le professionnel dans une posture lui permettant d'attendre patiemment la fin du cheminement d'une patiente

«muette». L'écoute des dernières paroles d'une personne en fin de vie, comme nous venons de le voir, nécessite énormément d'énergies, de temps et de patience; ce que les soignants arrivent à faire dépend indubitablement de l'organisation du travail et du temps imparti.

LES SOIGNANTS AURAIENT INTÉGRÉ, BIEN MALGRÉ EUX, LA NORME TEMPORELLE DONNÉE PAR L'ORGANISATION DE L'INSTITUTION, SI BIEN QU'UN PATIENT QUI ABANDONNE TROP TÔT, ARRIVE TROP TARD DANS LE SERVICE, OU DEMANDE À MOURIR OU ENCORE RÉSISTE, PERTURBERAIT LE RYTHME LÉGITIME DU CHEMINEMENT.

La planification du travail des infirmières, telle qu'elle est affichée dans la salle de soins, n'évoque pas le travail effectué après la mort d'un patient, un travail pourtant structurant à l'unité de soins palliatifs. Il présente essentiellement le travail de "préparation des prescriptions injectables », "vérification des stocks », «changes», "mobilisation», «soins d'hygiène et soins techniques » et "aide aux repas». Tout un pan des tâches postmortem, réservées aux soignants, est omis du planning officiel. Le programme ne fait pas mention du temps consacré auprès du défunt, alors qu'il est constitutif de la mission d'assistance aux mourants attribuée aux soins palliatifs. Comment comprendre qu'une institution délivrant une «bonne mort» omette de cette manière le temps de travail sur le cadavre? Une fois que le patient sombre dans le coma agonique, une autre activité commence pour les infirmières. Elles entament un travail de «maternage» (Castra, 2003, p. 170) et elles prennent soin de l'apparence du corps. Puis une fois que le décès intervient, elles préparent véritablement la présentation du corps du défunt: le minimum requis est la toilette, l'habillage et le maquillage. Les soignantes organisent l'arrivée des familles et décorent à cette fin le lit et la chambre. Elles se montrent alors compatissantes et apportent, à ce moment-là, davantage de réconfort aux familles. La mort du patient semble ouvrir le temps de l'entente entre l'institution et ses usagers. Les gestes, les attitudes à adopter après un décès et les consignes sont sues par les initiées, mais aucune trace ne transparaît dans les règles administratives. Faut-il en déduire que la compassion, l'attention au corps du défunt, sont des qualités innées pour les infirmières des soins palliatifs puisque l'institution n'éprouve pas le besoin de spécifier ces tâches?

Le temps de travail consacré à la toilette mortuaire et à l'accompagnement d'une famille endeuillée est prévu et anticipé dans l'organisation du travail des soignants, sauf qu'il apparaît sur le planning sous l'intitulé de «pause»- si bien que le jour où l'institution vit un décès, les soignants s'exclament: "On a du boulot!» E

\section{.}




\section{PRÉSENTATION DES TROIS « IM-PATIENTS}

\section{Histoire du patient A}

72 ans, femme, juive pratiquante, ancienne avocate, veuve.

Cette femme a été avocate au Liban où elle a vécu pendant plusieurs années. Ses enfants éprouvaient une grande admiration pour leur mère, présentée comme une femme pleine d'intelligence et de grâce. Elle a vécu avec son mari dans les «beaux » quartiers. Elle a eu trois enfants dont un médecin-chercheur en pharmacologie. Depuis le décès de son mari, un an plus tôt, la patiente souffrait d'un zona pour lequel elle a été traitée à l'hôpital. À la suite de ce traitement, les médecins ont diagnostiqué un cancer à un stade très avancé. Elle n'a pas suivi de traitement contre son cancer, et elle a obtenu une place dans l'unité de soins palliatifs. Ses proches la couvraient de fleurs et d'attention, sa chambre a été décorée avant son arrivée à l'hôpital. La patiente est arrivée très enthousiaste, elle souriait et se montrait très cordiale envers le personnel soignant. À ses premiers jours dans l'unité, la patiente avait espoir que le chef de service puisse la sauver. Après quelques jours, elle s'est résignée. Elle a refusé tous les traitements et a coupé toute relation de proximité avec le personnel hospitalier. Elle «s'est laissée mourir» (propos de l'équipe de l'unité).

\section{Histoire du patient B}

70 ans, homme, chrétien protestant, ancien chirurgien, marié.

Cet homme a été médecin chirurgien facial, dans un hôpital privé très réputé. Le patient a dû affronter des ruptures biographiques: sa femme a été internée en psychiatrie et l'un de ses enfants s'est suicidé. Il exprimait sa douleur pour la mort de son cadet. Un autre de ses fils est avocat. Le patient connaît le monde de la psychiatrie, ce qui pourrait expliquer en partie son refus de recevoir la psychologue. Le patient négociait la trajectoire avec le chef de service et il cherchait à savoir en détail l'évolution de son cancer du poumon. Ensuite, il a manifesté un refus de vivre l'agonie en prévenant le médecin qu'il « ne voulait pas être comme le monsieur d'en face [en état de coma agonique]» (propos du patient). À la fin de ses jours, malgré l'effet des produits antalgiques, le patient ne perdait pas pied avec la réalité: il suivait les informations à la télévision, son fils lui affichait ses rendez-vous planifiés sur son placard. Il était «dans la maîtrise», selon les infirmières: maîtrise de son temps et maîtrise de sa mort.

\section{Histoire du patient $\mathrm{C}$}

57 ans, homme, chrétien protestant, ancien aide-soignant de psychiatrie, marié.

Cet homme vivait dans les quartiers populaires. Il a eu deux femmes. La première fut la mère de ses deux premiers enfants. Elle est une employée du cimetière local. Sa seconde épouse travaillait à ses côtés, comme aide-soignante en psychiatrie. Comme le patient $\mathrm{B}$, il refusait de se confier à la psychologue et rejetait les analyses psychologiques. Le patient surprenait les soignants et le médecin par la lucidité avec laquelle il se confrontait à la réalité de sa mort. Il manifestait une violence contre l'institution en adressant des questions directes sur l'échéance de sa mort au médecin. Dès son arrivée, le patient insistait pour être euthanasié. Il a quitté l'unité et il est mort deux jours après, chez une cousine, en Bretagne «face à la mer, comme il l'a voulu» (propos de son épouse).

luttant jusqu'au bout et prolongeant ainsi la durée du processus de l'agonie. Par souci de clarté, nous avons opté pour une seule de ces deux figures.

Comme nous avons commencé à le voir plus haut, les soignants, tout comme le psychologue, se montrent plus pressés dans certaines circonstances. Les soignants auraient intégré, bien malgré eux, la mort et d'offrir une attitude «convenable », conforme aux attentes des soignants. Ceux qui désertent le devoir de vivre rompent en quelque sorte le «contrat implicite avec la société, qui est de ne pas mourir "de cette façon" »(Strauss, 1992, p. 119) sans avoir affronté la mort. De même, ceux qui essaient de survivre à leur mort sont aussi perçus par les professionnels comme des patients «difficiles». Un patient qui refuse les antalgiques et abandonne trop tôt (patient A), ou un patient qui s'insurge et demande à mourir dès sa première semaine (patient $\mathrm{B}$ et $\mathrm{C}$ ) tendent à accélérer le rythme du travail.

Leur résistance se traduit en partie par une «im-patience» à suivre la trajectoire de la «bonne mort». La figure du «mauvais» patient, comme nous l'avons désigné, recouvre une population d'usagers se reconnaissant à deux caractéristiques. Ils refusent de suivre le temps moyen du mourir et ils se montrent hostiles à toute forme d'analyse de nature psychologique. Par ailleurs, il se trouve que les trois cas «im-patients» rencontrés et décrits ici se caractérisent, chacun à leur manière, par une proximité sociale plus ou moins forte avec le monde médical et par une absence de socialisation à la religion catholique.

Dans le cas du patient A, l'«im-patience» se traduit par une déprise précoce par rapport au pronostic des soignants. Avant que madame A choisisse de se "laisser mourir», son fils aîné, médecin, posait une limite à la prescription de morphine. Il ne partageait pas la politique pharmacologique du chef de service. À travers le jeu de négociation autour des prescriptions, l'un limitant et l'autre prescrivant, les deux médecins ne poursuivaient pas la même idée du processus du mourir. L'un, pris dans sa position filiale et affective, estimait pouvoir retarder l'instant de la mort de sa mère, tandis que l'autre, pris dans sa position professionnelle, anticipait le soulagement de ses douleurs. Ces deux logiques temporelles étaient en confrontation, et la patiente, après plusieurs jours d'évitement des soignants, a cédé en «se laissant mourir ».

Dans les deux autres cas présentés (B et C), l'«im-patience» se traduit par une demande de mort adressée directement aux médecins. Dans ces deux cas, l'«impatient» détient une connaissance sur le monde médical avec lequel il a été plus ou moins socialisé. Ces patients connaissent suffisamment bien les règles pour pouvoir tenter de les détourner. Leur position sociale leur donne le pouvoir de discuter la ligne politique du service. Ils sont euxmêmes porteurs d'une connaissance sur leur corps, et partagent aussi le langage des autres soignants. Leur ancien statut de 
soignant dans un cas et de médecin dans l'autre leur donne, en effet, les armes pour exiger des soignants des réponses concrètes. Non seulement ces patients se montrent exigeants, mais ils souhaitent savoir combien de temps il leur reste à vivre et demandent à mourir si le temps leur paraît long (cas de $\mathrm{B}$ et de $\mathrm{C}$ ). Grâce à leur ancien statut de professionnel, ils maîtrisent le discours de l'institution médicale et cela leur permet de refuser l'analyse de nature psychologique.

Voici un extrait d'entretien entre le chef de service $(\mathrm{CdS})$ et le patient qui était précédemment un chirurgien (B) :

B : «Bon, je sais ce que j'ai... on est d'accord, on est en phase terminale. Je vois le monsieur en face [un autre patient était dans le coma dans une chambre voisine], je ne veux pas finir comme ça, alors si on pouvait accélérer le processus...»

CdS : "Mais on ralentit le processus, on n'accélère pas.»

Le jour suivant, le chef de service (CdS) est accompagné d'une collègue anesthésiste (An). Le patient répétait en étant plus provocant:

B : "Bon comment ça va se passer quand je serai dans le trou?!» [aucune réponse]

An : «On n'a pas le droit d'enlever la vie. » [le patient ne répond pas]

An: "Mais, vous êtes père ${ }^{10}$ ! [silence] Voulez-vous qu'on vous rajoute quelque chose pour vous... détendre?»

B : «Non! ça va comme ça. Je ne suis pas "angoissé"! [le patient montre qu'il partage le langage des médecins] Je suis TRÈS BIEN avec ce que j'ai! »

CdS : "C'est bien, vous vous posez des questions sur l'avenir, mais vous devriez vivre les instants présents» $[\ldots]$ «ne vous posez pas tant de questions, vivez au jour le jour... pour l'instant, vous n'avez pas de souci à vous faire. Il ne devrait pas y avoir de problème. »

B: «Non, mais je me pose des questions... je ne peux plus me lever...»

CdS : «Pour l'instant, vous êtes FATIGUÉ! [il accentue sa voix en invoquant la raison de la "fatigue". De cette manière il insinue au patient qu'il devrait "se calmer" et que la fatigue explique sa faiblesse physique. En réalité, le processus du mourir est bien entamé], et vous devriez conserver votre énergie [à penser à autre chose qu'à la mort...]»

Les deux médecins ne s'attardent pas dans la chambre, ils sortent. L'entretien est interrompu.

Le patient résiste à l'analyse psychologique des deux médecins. Malgré une proximité sociale entre les trois protagonistes, la mauvaise volonté à s'adapter aux soins prodigués contraint le patient à une sérieuse mise à l'écart. Cet élan précipité vers la mort est incompris par les médecins du service. Le chef de service montre une forme d'agacement vis-à-vis de l'ancien confrère qui ne suit pas la politique du bien mourir. Les phases de silence rompant abruptement l'entretien rendent bien compte de la violence de l'échange et du choc ressenti par les soignants. Les médecins semblent déconcertés quand ils sont confrontés à ce type de demande qui manifeste une violence à l'égard de l'institution. Les médecins auraient donc moins de ressources face à un patient socialisé au monde médical, et qui partage l'état de connaissance sur la maladie et le pronostic. Ici, ils tentent en vain de le raisonner.

\section{UN TRAVAIL SOCIAL DE DÉCRYPTAGE DES ÉMOTIONS DES «IM-PATIENTS »}

Comment l'institution s'y prend-elle pour réduire ainsi le trouble produit par les «im-patients»? Le souci de l'institution médicale d'être à l'écoute des besoins des patients amène parfois les professionnels des soins palliatifs à faire face à des paroles violentes de demandes de mort. Les soignants acceptent, certes, beaucoup des patients, mais à condition que cela n'engendre pas un remaniement profond de la norme temporelle de la «bonne mort». L'«im-patient» risque d'être stigmatisé lors des transmissions par les soignants, et d'être étiqueté comme "angoissé», «exigeant» ou «demandeur».

Reprenons le cas de l'ancienne avocate (patient A). Alors qu'elle exerçait une profession dont la particularité était paradoxalement d'user de la parole pour convaincre, elle s'est progressivement enfermée dans le mutisme. Pourquoi un tel revirement alors qu'elle était si enjouée à son arrivée? La patiente ne s'exprime plus et ne communique plus avec les soignants après que son fils aîné, médecin, eut montré son désaccord quant à l'usage de morphine avec les médecins du service. Non seulement la patiente refuse le dialogue avec les soignants, mais de surcroît elle n'avale plus les pilules de morphine prescrites. Elle suit l'avis de son fils pharmacologue et contrarie ainsi le travail des soignants du service. L'abandon prématuré d'un patient «mutique» est injustifié aux yeux des soignants, étant donné qu'il reçoit des soins de confort et ne montre plus de signes apparents de souffrance. Les soignants mis en échec dans le processus de la «bonne mort» limitent leurs interventions, malgré la position sociale privilégiée de la patiente ${ }^{11}$. Les soignants font le maximum pour maîtriser les douleurs physiques et luttent avec l'aide du patient pour supprimer les souffrances. Ils ne peuvent guère faire plus et estiment que seul le patient doit trouver, en lui, ce qu'ils appellent $l a$ force mentale, une force nécessaire pour faire face à la situation. Un soir, en refermant la porte de la chambre de la patiente A, arrivée depuis quinze jours dans l'unité, un infirmier nous confie manifestement déçu: "elle se laisse mourir... ce n'est pas bien... elle n'a pas de force mentale pour survivre». Parlant d'une seule voix, l'équipe juge qu' elle ne fait pas beaucoup d'efforts ». Quand les soignants entrent dans la chambre de cette patiente, elle ne sourit plus comme aux premiers jours. Elle reste béate, un peu abattue et indifférente aux gestes des soignants. En recevant tous les soins de confort, elle aurait dû être en mesure d'atteindre l'état de sublimation.

Il arrive, comme dans le cas A, que les patients refusent de confier leurs émotions sur leur mort à des soignants qu'ils connaissent depuis peu. Rester ainsi dans le mutisme peut être, à l'origine, un besoin de protéger son intimité. Ne pas se confier ne signifie pas d'emblée que le patient oppose un refus de coopérer. Or, c'est bien de cette manière que les soignants interprètent ce comportement. Confrontée à une réaction de mutisme, l'invitation à s'exprimer prévue par le dispositif des soins palliatifs se montre plus pressante. Ils retardent l'entrée dans la chambre ou n'y entrent qu'au moment nécessaire pour donner les soins et privilégient les moments où elle est endormie pour éviter tout contact. Ils substituent à leur compassion l'éloignement. Pour les soignants, ce type de patient n'a pas su affronter la mort, ce qui au fond les déçoit: les médecins baissent la tête, ainsi que les infirmiers, en parlant de son décès. Le chef de service perçoit sa mort comme un échec de l'accompagnement, ce qu'il exprime par un soupir: "elle s'est laissée mourir». Isolée et refermée sur ellemême, la patiente refuse la «bonne mort» délivrée par l'institution, et est conduite, isolée, à se laisser mourir.

Le décryptage de ces signes d'angoisse diverge quelquefois entre deux soignants. Leurs interprétations s'ajustent au moment d'échanger leurs perceptions et de décider des protocoles de soins. Les soignants se comprennent et utilisent un même vocabulaire: "protocole douleur», «protocole angoisse», "protocole agitation », «protocole dyspnée», "protocole encombrement »... D'où l'intérêt de savoir s'il s'agit bien d'une angoisse, d'une agitation ou d'une douleur. Un problème d'interprétation se pose quant aux signes saisissables du «dehors». Comme l'écrivait F. Laplantine: «tout ce qui concerne 
les affects et les sentiments éprouvés "du dedans" est souvent rigoureusement irréductible à ce qui peut être lu "du dehors" » (Laplantine, 2003, p. 17). Concentronsnous sur la lecture du «dehors » entreprise en premier lieu par les infirmières. Les soignantes, en grande majorité des femmes, sont les plus présentes au chevet du patient et elles suivent intégralement la trajectoire du patient, ce qui n'est pas toujours le cas du reste de l'équipe. À ce titre, elles bénéficient d'une place privilégiée. Elles se font «naturellement» les porteparole légitimes des patients concernant leurs goûts et leurs habitudes, et ce, même s'ils sont «muets » dans leur agonie. C'est donc une lecture féminine des maux qui est produite concernant le processus de mort en unité de soins palliatifs en privilégiant une écoute pour une fin de vie continue, lente et entourée.

Les attitudes de refus du dispositif arrangé par l'institution sont interprétées comme un problème psychologique, et cela avant même que le psychologue n'ait vu le patient pour le constater. Le patient «lui-même est [...] suspecté d'être aveuglé par sa subjectivité » (Lascoumes, 2002, p. 121), alors qu'elle est si sollicitée par les soignants (Memmi, 2003). Le décryptage des paroles de l'«im-patient» révèle une stratégie des soignants visant à éloigner une norme temporelle concurrente. L'institution offre calmement aux «im-patients» des paroles rassurantes. Le médecin, ainsi que l'équipe solidaire de la position de l'institution, résiste face à une demande de mort. Ils poursuivent leur démarche de soins et l'éducation du patient sur le sens de la «bonne mort». De cette manière, l'institution, à travers l'intervention du médecin, s'obstine à guider le patient par des "points de repères aussi simples que: "on continue de prendre soins de... on ne vous abandonne pas", "je prendrai soin de vous jusqu'au bout", "je ne ferai rien pour hâter votre mort", et "je continue de faire tout ce qui est possible et nécessaire pour vous soulager" ${ }^{12}$. Le refus de lutter pour survivre de certains patients, ou celui de leurs proches rompt donc le tracé d'une «bonne mort» définie par les soins palliatifs.

Au terme de cet article, on peut souligner deux points. D'une part, l'idée d'une «bonne mort» comprend une durée idéale, ni trop courte, ni trop longue. Elle est le fruit d'un compromis entre l'état de santé des patients et une organisation rationalisée du travail. Présentée comme une durée raisonnable, la moyenne des « 21 jours» sert de référence pour évaluer la bonne réalisation du mourir. D'autre part, notre travail a montré que l'idéal de la «bonne mort» est parfois confronté à une lecture distante de certains patients que nous avons désignés sous l'étiquette d'«im-patients »; une lecture distante à l'égard de la temporalité de l'organisation de la mort et de l'usage de la psychologie en milieu hospitalier. Ces «im-patients» tentent d'accélérer le rythme d'une mort présentée comme «naturelle»par les agents de l'institution.

Enfin, dans une prochaine étude, il faudrait savoir si l'analyse proposée à partir des trois cas rencontrés lors de notre ethnographie, cas qui se caractérisent par leur socialisation au monde médical et leur distance au catholicisme, peut être appliquée à l'ensemble de la population des «im-patients » accueillis dans les unités de soins palliatifs.

\section{Bibliographie}

ABIVEN, M. (1997). Une mort plus humaine: expérience d'une unité hospitalière de soins palliatifs, Paris, InterÉditions.

ANONYME (2007). «La politique des soins palliatifs », Rapport de la Cour des comptes, Paris, février 2007, p. 349-381.

CASTRA, M. (2003). Bien mourir: Sociologie des soins palliatifs, Paris, PUF.

GLASER, B.G. et A. STRAUSS (1968). Time for Dying, Chicago, Aldine.

GLASER, B.G. et A. STRAUSS, (1965). Awareness of Dying, Chicago, Aldine.

HERZLICH, Cl. (1976). "Le travail de la mort», Annales - Économie, Sociétés, Civilisations, Paris, $\mathrm{n}^{\circ}$ 1, p. 197-217.

KÜBLER-ROSS, E. (1997 [1981]). Vivre avec la mort et les mourants, Paris, Du Tricorne.

LAPLANTINE, F. (2003 [1992]). Anthropologie de la maladie. Étude ethnologique des systèmes de représentations étiologiques et thérapeutiques dans la société occidentale contemporaine, Paris, Payot.

LASCOUMES, P. (2002). «Représenter les usagers ", dans I. BASZANGER et al., Quelle médecine voulons-nous?, Paris, La Dispute, p. 107-125.

MEMMI, D. (2003). Faire vivre et laisser mourir, le gouvernement contemporain de la naissance et de la mort, Paris, La Découverte.

OLLIVIER, F. (2004). 21 jours en moyenne. Regard sociologique sur l'unité de soins palliatifs, mémoire de DEA de sociologie, sous la direction de Dominique MEMMI et Olivier SCHWARTZ, Université Descartes, Paris.

STRAUSS, A. (1992). La trame de la négociation: sociologie qualitative et interactionnisme, textes réunis par Isabelle BASZANGER, Paris, L'Harmattan.

\section{Notes}

1. L'auteure tient à remercier les premiers lecteurs de ce texte: D. Memmi, pour ses précieux conseils et sa lecture; I. Gouarné, pour son soutien; E. Taïeb, E. Requilé, et D. Müller, pour la pertinence de leurs critiques.

2. Les chercheurs en sciences sociales ont beaucoup publié sur le thème de la mort à l'hôpital. Nous pensons ici à E. Morin (1970), Louis Vincent Thomas (1975) et Philippe Ariès (1975). La revue Annales a publié un numéro spécial «autour de la mort» (1976).

3. La circulaire de la DGS du 26 août 1986 relative à l'organisation des soins et à l'accompagnement des malades en phase terminale est plus connue sous le nom de l'auteure engagée Geneviève Laroque.

4. Le rapport montre que les ressources attribuées aux structures de soins palliatifs pour les établissements de santé publics et privés décroissent progressivement de 11,43 millions d'euros en 2002 à 8,13 millions d'euros en 2007. Le même rapport démontre un affaiblissement des ressources financières au profit de leur intégration dans le financement du plan cancer.

5. Nous devons préciser que le présent article a vocation d'exposer des pistes d'une recherche en cours.

6. Les données présentées proviennent d'une thèse en cours de réalisation. Plus de détails sur la méthodologie de l'enquête sont donnés dans mon mémoire de DEA (voir Ollivier, 2004). La durée d'un mois, renouvelée deux fois, fut le temps maximal convenu par chacun des chefs de service pour ma présence sur le terrain.

7. Les expressions en italique, dans le corps du texte, proviennent du vocabulaire indigène.

8. Il s'agit ici de bénévoles, non tout à fait profanes. Ces derniers sont recrutés, puis ils sont soumis à une formation initiale de 90 jours, période pendant laquelle ils suivent une « formation théorique » de trois jours, un parrainage de trois mois et une formation pratique de deux jours.

9. Propos du psychologue recueillis lors d'un stage d'observation en unité de soins palliatifs.

10. L'anesthésiste fait ici référence aux enfants de ce patient pourtant absents au moment de la consultation.

11. Le fils aîné était médecin-pharmacologue. Cet élément biographique fut très vite annoncé lors des réunions de l'équipe soignante.

12. Les propos sont issus d'un entretien conduit avec un médecin de soins palliatifs, confronté à la demande de mort (cas du patient $\mathrm{C}$ ). 